\title{
Association Between Vertebral Segmental Artery Occlusion and Kummell Disease: A Prospective Cohort Study Based On MRA
}

\section{Sizheng Zhan}

Peking University People's Hospital

Tianyu Zhang

Peking University People's Hospital

\section{Wenyong Xie}

Peking University People's Hospital

Feng Xue ( $\sim$ pku_xf@163.com )

Peking University People's Hospital

Dianying Zhang

Peking University People's Hospital

\section{Baoguo Jiang}

Peking University People's Hospital

\section{Research article}

Keywords: Kummell disease, vertebral compression fractures, vertebral segmental artery, MRA, vertebroplasty

Posted Date: October 13th, 2021

DOl: https://doi.org/10.21203/rs.3.rs-952409/v1

License: (c) (i) This work is licensed under a Creative Commons Attribution 4.0 International License. Read Full License 


\section{Abstract \\ Purpose}

We designed a prospective cohort study based on magnetic resonance angiography (MRA) to identify whether the degree of Kummell disease (KD) vertebral artery occlusion is more severe than that of simple vertebral compression fractures.

\section{Methods}

We enrolled elderly patients with VCFs who met the established criteria from January 2019. MRA was used to determine the degree of vertebral artery occlusion. We defined the lesion segmental occlusion rate (LSOR) as the sum of the ischemic values of the bilateral vertebral segmental arteries ( 0 means expedite, 1 means stenosis, 2 means occlusion; range from $0-4$ ). The average LSOR is the sum of LSORs divided by the number of vertebrae. Follow-up outcomes included VAS and ODI scores after surgery. X-rays were re-examined at 1 year after surgery to determine whether the vertebral body had recollapsed.

\section{Results}

25 cases of KD segments and 37 cases of non-KD segments were included. The average LSOR in KD segments was significantly higher than that in non-KD segments ( 1.44 vs $0.32, P<0.01)$. The recollapse rate of the KD segments after one year was significantly higher than that in non-KD segments ( $56 \% \mathrm{vs}$ $27 \%, P=0.03)$. In non-KD segments, $57.1 \%$ of segments with a high LSOR $(1-2)$ recollapsed, and $20 \%$ of segments with a low LSOR $(0)$ recollapsed $(P=0.045)$

\section{Conclusions}

The degree of artery occlusion in the KD segment is more serious than that in the non-KD segment. KD segments or non-KD segments with a high degree of artery occlusion will have a higher recollapse rate.

\section{Summary}

Through a designed prospective cohort study based on MRA, we identified that the degree of segmental artery occlusion in the KD is more serious than that in the non-KD segments. We have verified that the destruction of the blood supply is an important factor in the formation of KD.

\section{Introduction}

Kummell disease (KD) is a late complication of osteoporotic vertebral compression fractures (VCFs) ${ }^{[1]}$, first described by Hermann Kummell in $1895^{[2]}$. KD manifests as low back pain, neurological deficits, or 
kyphosis that occur several weeks or months after an asymptomatic period after minor trauma ${ }^{[3]}$. It is characterized by collapse of the vertebral body and intravertebral cleft (IVC) after trauma ${ }^{[4]}$. The complications of recollapse ${ }^{[5,6]}$ and bone cement leakage $\mathrm{e}^{[7-9]}$ after vertebroplasty in KD patients are significantly increased, than that of simple VCFs.

Currently, there are many hypotheses about the pathogenesis of $\mathrm{KD}^{[10]}$, including ischemic osteonecrosis $^{[11-13]}$, air formation ${ }^{[14,15]}$, changes of bone biomechanics ${ }^{[16,17]}$ and some other hypotheses with supportive clinical or biomechanical evidence ${ }^{[10]}$. Among them, the hypothesis of ischemic osteonecrosis is the most studied hypothesis and recognized by most researchers ${ }^{[10]}$. Researchers believe that the formation of IVC is caused by direct factors, including fat embolism or fracture compression, or secondary factors, such as corticosteroid use, alcoholism, and vasculitis, resulting in the destruction of the blood supply of the vertebral body after the initial minimal trauma. The vertebral body gradually undergoes osteonecrosis, and the vertebral body collapses, which further destroys the blood supply of the vertebral body, forming a vicious cycle that eventually progresses to $\mathrm{KD}^{[18]}$.

Lin et al. ${ }^{[13]}$ reported that in patients with osteoporotic VCFs before vertebroplasty, decreased bone marrow perfusion, as measured by dynamic contrast-enhanced magnetic resonance imaging (DCE-MRI), was associated with IVC formation. Kim et al. ${ }^{[12]}$ found that most patients with IVC have unilateral or bilateral segmental artery occlusion through magnetic resonance angiography (MRA). These studies provided evidence for the hypothesis of vertebral avascular necrosis. However, these studies were singlearm observational studies. We designed a prospective cohort study based on MRA to identify whether the degree of KD vertebral artery occlusion is more severe than that of simple VCFs.

\section{Methods}

We enrolled elderly patients with VCFs who met the established criteria from January 2019. The study was approved by the ethics committee of Peking University People's Hospital, number 2019PHB240. All patients had the contents of the study fully explained and signed the informed consent form before enrollment.

\section{Inclusion and Exclusion Criteria}

\section{Inclusion Criteria}

- Were $\geq 65$ years old;

- Diagnosed as fresh VCFs by preoperative MR (ranging from T10-L4);

- Had complete X-ray, CT, MRI, and MRA examinations before surgery;

- Underwent vertebroplasty.

\section{Exclusion Criteria}


- Fractures caused by infection or malignant tumors;

- Imaging examinations or physical examinations suggesting spinal cord compression;

- Inability to tolerate surgery;

- Inability to cooperate to complete the required follow-up period.

\section{Data Extraction and definition}

The characteristics of the patients were recorded, including age, sex, body mass index (BMI), and preoperative medical history that may be related to the formation of IVC, such as smoking, alcohol abuse, glucocorticoid use and diabetes. The time interval between the development of back pain to the time of MRA, fracture segment, preoperative visual analog scale (VAS) score and Oswestry disability index (ODI) score were recorded.

Follow-up outcomes included VAS and ODI scores at 1 week, 1 month, and 12 months after surgery. Xrays were re-examined at 1 year after surgery to calculate the vertebral compression rate (VCR) and kyphotic angle (KA) to determine whether the vertebral body had recollapsed.

We defined the lesion segmental occlusion rate (LSOR) as the sum of the ischemic values of the bilateral vertebral segmental arteries ( 0 means expedite, 1 means stenosis, 2 means occlusion; range from $0-4$ ). The average LSOR is the sum of LSORs divided by the number of vertebrae. We defined the normal vertebra as no fracture observed by MRI at the time of enrollment, and no collapse found on X-ray reexamination after 1 year.

The diagnosis of KD requires a comprehensive analysis of the patient's medical history, clinical symptoms, and characteristic imaging findings, as shown in Figure 1.

\section{Radiological Assessment}

Two authors individually and independently assessed the imaging data twice to eliminate intraand interobserver bias. If there was a noticeable difference in any result, the two authors and a senior doctor conferred to determine the final result. We defined VCR $=1$ - the height of the maximum compression of the vertebral body $* 2$ / (anterior edge height of vertebral body + posterior edge height of vertebral body), as shown in Figure 2. We defined the KA as the angle formed between the cranial endplates and the caudal endplates of the vertebral body. Two conditions were defined as a recollapse of

the vertebral body ${ }^{[6,19]}$ : $(1) \geq 15 \%$ progression of VCR between the immediately postoperative and last follow-up period; $(2) \geq 10^{\circ}$ progression in local KA between the immediately postoperative and last follow-up period.

\section{Statistical Analysis}

All analyses were performed using IBM SPSS Statistics for Windows, version 26.0 (IBM Corp., Armonk, N.Y., USA). Between-group comparisons of variables were performed by chi-square tests and Fisher's 
exact tests, whereas between-group comparisons of continuous variables were performed by t-tests or Mann-Whitney U tests.

\section{Results}

\section{Characteristics of the population}

A total of 55 patients and 62 thoracolumbar or lumbar fracture segments (T10-L4) were included in the study, including 25 cases of KD segments and 37 cases of non-KD segments. There was no significant difference between the two groups in terms of age, sex, BMI or risk factors for IVC formation, as shown in Table 1.

Table 1

Characters of patients.

\begin{tabular}{|c|c|c|c|}
\hline & $K D(n=25)$ & Non-KD $(n=37)$ & P-value \\
\hline \multirow[t]{2}{*}{ Age (mean $\pm S D)$} & & & 0.12 \\
\hline & $75.6 \pm 4.2$ & $73.8 \pm 4.4$ & \\
\hline \multicolumn{4}{|l|}{ Gender (female/male) } \\
\hline & $15 / 10$ & $23 / 14$ & \\
\hline \multirow[t]{2}{*}{ BMI (kg/m2) } & & & 0.67 \\
\hline & $23.9 \pm 3.9$ & $23.5 \pm 3.5$ & \\
\hline Risk factors of IVC & & & 0.95 \\
\hline Smoking & 3 & 4 & \\
\hline Alcohol consumption & 3 & 5 & \\
\hline Glucocorticoids & 1 & 1 & \\
\hline Diabetes & 2 & 4 & \\
\hline \multirow[t]{2}{*}{ Time to MRA (days) } & & & $<0.01^{*}$ \\
\hline & $36.1 \pm 41.6$ & $8.7 \pm 5.3$ & \\
\hline \multirow[t]{2}{*}{ Average LSOR } & & & $<0.01^{\star}$ \\
\hline & 1.44 & 0.32 & \\
\hline \multirow[t]{2}{*}{ Recollapse Rate } & & & $0.03^{*}$ \\
\hline & $56 \%$ & $27 \%$ & \\
\hline
\end{tabular}




\section{Results of MRA}

In normal vertebra, the average LSOR was 0.27 . For KD segments, the LSOR of each segment were categorized as $0(9 / 25), 1-2(11 / 25)$, and $3-4(5 / 35)$. The average LSOR was 1.44 . In the non-KD segments, the LSOR of each segment were categorized as 0 (30/37) and 1-2 (7/37). The average LSOR was 0.32 . We found that there was a significant difference in the average LSOR between KD and non-KD segments $(P<0.01)$, and there was no simultaneous occlusion of the bilateral vertebral segmental arteries in non-KD segments.

\section{Postoperative results}

The recollapse rate of the KD segments after one year was $56 \%$, and the recollapse rate of the non-KD segments was $27 \%(P=0.03)$. In non-KD segments, $57.1 \%$ of segments with a high LSOR $(1-2)$ recollapsed, and $20 \%$ of segments with a low LSOR $(0)$ recollapsed $(P=0.045)$. At the final follow-up, there was no significant difference in the VAS and ODI scores between the KD and non-KD groups, as shown in Table 2 .

Table 2

Comparisons of VAS score and ODI score between KD and non-KD.

\begin{tabular}{|clllll|}
\hline Group & Preoperation & $\begin{array}{l}\text { 1 week } \\
\text { postoperation }\end{array}$ & $\begin{array}{l}\text { 1 month } \\
\text { postoperation }\end{array}$ & $\begin{array}{l}\text { 1 year } \\
\text { postoperation }\end{array}$ \\
\hline VAS & KD & $7.5 \pm 2.0$ & $3.4 \pm 1.6$ & $2.7 \pm 1.2$ & $1.4 \pm 0.8$ \\
\hline $\begin{array}{l}\text { Non- } \\
\text { KD }\end{array}$ & $7.3 \pm 1.1$ & $4.4 \pm 1.1$ & $2.3 \pm 1.12$ & $1.6 \pm 1.1$ \\
\hline $\begin{array}{l}\text { P- } \\
\text { value }\end{array}$ & 0.35 & 0.25 & 0.42 & 0.38 \\
\hline ODI & KD & $66.3 \pm 15.1$ & $39.6 \pm 19.2$ & $33.6 \pm 16.2$ & $16.1 \pm 12.0$ \\
\hline $\begin{array}{l}\text { Non- } \\
\text { KD }\end{array}$ & $73.1 \pm 14.9$ & $43.2 \pm 10.0$ & $40.3 \pm 15.1$ & $18.4 \pm 11.1$ \\
\hline $\begin{array}{l}\text { P- } \\
\text { value }\end{array}$ & 0.171 & 0.36 & 0.24 & 0.35 \\
\hline
\end{tabular}

\section{Other findings}

The average time interval between the development of back pain to the time of MRA in the KD group was 35.6 days compared with the average time interval in the non-KD group of 9.1 days $(P<0.01)$. The course of KD was categorized as 1 week (10/25), 1 month (7/25), 3 months (3/25) and more than 3 months (5/25). The average LSOR was 2.71 (within 1 week), 1.00 (within 1 month), 0.60 (within 3 months), and 1.67 (over 3 months), respectively.

\section{Discussion}


To our knowledge, this is the first prospective cohort study of segmental artery occlusion in KD. Our results suggest that the degree of occlusion of KD segmental arteries was significantly higher than that of non-KD segmental arteries. Fractured segments with a high degree of artery occlusion had a significantly higher probability of recollapsing after vertebroplasty. There was no significant difference in postoperative VAS and ODI scores between KD and non-KD patients.

There are many hypotheses about the pathogenesis of $\mathrm{KD}$ formation ${ }^{[4,11,12]}$, among which ischemic osteonecrosis is the most widely supported and studied hypothesis ${ }^{[10]}$. Avascular osteonecrosis after VCFs has its own anatomical theory. Ratcliffe $\mathrm{e}^{[20]}$ used microangiography to study the arterial anatomy of the adult lumbar vertebral body and proposed that the upper and lower anterior segments of the vertebral body are the watershed areas of the vertebral body. These areas are susceptible to ischemia. Prakash ${ }^{\text {[21] }}$ also reported that the central anterior branch of the pair of segmental arteries supplies the ventral side of one vertebral body, while the central posterior branch supplies the dorsal side of two adjacent vertebral bodies. Therefore, the dorsal side of the vertebral body receives collateral blood flow, but the ventral side does not; this will theoretically make the ventral part of the vertebral body have a higher risk of blood supply destruction.

Lin et al. ${ }^{[13]}$ reported that in patients with osteoporotic VCFs before vertebroplasty, decreased bone marrow perfusion, as measured by DCE-MRI, was associated with IVC formation. Kim et al. ${ }^{[12]}$ found that most patients with IVC have unilateral or bilateral segmental artery occlusion through MRA. In our study, compared with normal vertebral bodies and simple VCFs, we found that the degree of KD segmental artery occlusion was significantly increased. All these results suggest that the destruction of blood supply may be an important reason for the formation of IVC, indicative of KD.

In many research reports, IVC has been suggest to be an important high-risk factor for recollapse after vertebroplasty ${ }^{[9,19,22]}$. Our results suggest that the recollapse rate of KD segments was significantly higher than that of non-KD segments. At the same time, the recollapse rate of VCFs with a high degree of artery occlusion in non-KD segments was also significantly increased. Therefore, we believe that in addition to IVC being a high-risk factor for recollapse, segmental artery occlusion is also an important high-risk factor for recollapse after vertebroplasty.

At present, the main treatment for KD is vertebroplasty ${ }^{[23-25]}$. Complications such as recollapse after vertebroplasty are relatively high in patients with $\mathrm{KD}$, and therefore, some clinicians have tried to use short-segment vertebral fixation to treat $\mathrm{KD}^{[26-28]}$. Our research suggests that there was no significant difference in VAS and ODI scores between KD and non-KD patients. This is also consistent with the results of many clinical reports that vertebroplasty is an effective treatment for $\mathrm{KD}^{[23-25]}$. There are also studies comparing the prognosis of vertebroplasty and short-segment vertebral fixation, and the results showed no significant differences in VAS and ODI scores ${ }^{[26-28]}$. We found that no non-KD segments progressed to KD after surgery, regardless of the degree of segmental artery occlusion. This may be 
because vertebroplasty restores the stability of the vertebral body, disrupts the vicious cycle mechanism leading to KD formation, and avoids the further blood supply damage.

It takes an amount of time for VCF to progress to $\mathrm{KD}^{[13]}$, and the time duration is currently inconclusive. Our findings suggest that the average course of disease in KD patients was significantly higher than that in non-KD patients. We also had a pretty interesting finding. The average LSOR of KD patients within 1 week, 1 month, 3 months, and over 3 months were 2.71, 1.00, 0.60, and 1.67, respectively. The average LSORs within 1 week and more than 3 months were significantly higher than those in the other two stages. For the short course within 1 week, we believe that it is precisely because of the high occlusion rate that VCF rapidly progresses to KD. Regarding the long course of more than 3 months, due to the long course of the disease, the degree of compression of the vertebral body increased, destroyed the blood supply and resulted in aggravation of segmental artery occlusion. Therefore, the destruction of blood supply and vertebral compression fracture are mutually promoting and vicious cycle processes that ultimately produce KD.

\section{Limitations}

First, although the number of target segmental arteries was obtained, the number is still small. Second, we could not determine the exact time or sequence of artery occlusion and formation of KD. This would require multiple MRA inspections from the initial time of injury, but this is difficult to achieve.

\section{Conclusion}

The initial damage or fracture progression insults the segmental arteries, which eventually leads to osteonecrosis and progresses to KD. Therefore, the degree of artery occlusion in the KD segment is more serious than that in the non-KD segment. At the same time, KD segments or non-KD segments with a high degree of artery occlusion will have a higher recollapse rate, indicating segmental artery occlusion is also an important high-risk factor for recollapse after vertebroplasty. For KD segments without nerve compression, vertebroplasty is still an effective treatment.

\section{Declarations}

\section{Funding}

This study was funded by Ministry of Education Key Laboratory of trauma treatment and nerve regeneration.

\section{Conflicts of interest}

SZ.Z, TY.Z, F.X, DY.Z and BG.J declare that they have no conflicting interests

\section{Availability of data and material}


The datasets generated and analyzed during the current study are not publicly available due to the data also forms part of an ongoing study but are available from the corresponding author on reasonable request

\section{Code availability}

Not Applicable

\section{Authors' contributions}

DY.Z and BG.J were responsible for the study design. SZ.Z, TY.Z and WY.X contributed to the acquisition and analysis of data. TY.Z, SZ.Z and F,X contributed to the interpretation and analysis of data. SZ.Z and WY.X were responsible for drafting the article. TY.Z and F.X were responsible for revising it critically for important intellectual content. DY.Z and BG.J have approved the version to be published and all subsequent versions. SZ.Z, TY.Z and WY.X were major contributors and should be listed as to co-first authors.

\section{Ethics approval}

The study was approved by the ethics committee of Peking University People's Hospital, number 2019 PHB240.

\section{Consent to participate}

All patients had the contents of the study fully explained and signed the informed consent form before enrollment.

\section{Consent for publication}

Not Applicable

\section{References}

1. Ranjan M, Mahadevan A, Prasad C, Sommana S, Susarla Krishna S. Kümmell's disease - uncommon or underreported disease: A clinicopathological account of a case and review of literature. Journal of neurosciences in rural practice. 2013;4(4):439-42. doi:10.4103/0976-3147.120234.

2. Brower AC, Downey EF Jr. Kümmell disease: report of a case with serial radiographs. Radiology. 1981;141(2):363-4. doi:10.1148/radiology.141.2.7291557.

3. Park SJ, Kim HS, Lee SK, Kim SW. Bone Cement-Augmented Percutaneous Short Segment Fixation: An Effective Treatment for Kummell's Disease? J Korean Neurosurg Soc. 2015;58(1):54-9. doi:10.3340/jkns.2015.58.1.54.

4. Li H, Liang CZ, Chen QX. Kümmell's disease, an uncommon and complicated spinal disorder: a review. J Int Med Res. 2012;40(2):406-14. doi:10.1177/147323001204000202. 
5. Heo DH, Chin DK, Yoon YS, Kuh SU. (2009) Recollapse of previous vertebral compression fracture after percutaneous vertebroplasty. Osteoporosis international: a journal established as result of cooperation between the European Foundation for Osteoporosis and the National Osteoporosis Foundation of the USA 20 (3):473-480. doi:10.1007/s00198-008-0682-3.

6. Yu WB, Jiang XB, Liang D, Xu WX, Ye LQ, Wang J. (2019) Risk factors and score for recollapse of the augmented vertebrae after percutaneous vertebroplasty in osteoporotic vertebral compression fractures. Osteoporosis international: a journal established as result of cooperation between the European Foundation for Osteoporosis and the National Osteoporosis Foundation of the USA 30 (2):423-430. doi:10.1007/s00198-018-4754-8.

7. Tanigawa N, Kariya S, Komemushi A, Tokuda T, Nakatani M, Yagi R, Sawada S. Cement leakage in percutaneous vertebroplasty for osteoporotic compression fractures with or without intravertebral clefts. AJR American journal of roentgenology. 2009;193(5):W442-5. doi:10.2214/ajr.09.2774.

8. Zhong BY, He SC, Zhu HD, Pan T, Fang W, Chen L, Guo JH, Deng G, Zhu GY, Teng GJ. Nomogram for Predicting Intradiscal Cement Leakage Following Percutaneous Vertebroplasty in Patients with Osteoporotic Related Vertebral Compression Fractures. Pain Physician. 2017;20(4):E513-20.

9. Ding J, Zhang Q, Zhu J, Tao W, Wu Q, Chen L, Shi P, Zhang H. Risk factors for predicting cement leakage following percutaneous vertebroplasty for osteoporotic vertebral compression fractures. European spine journal: official publication of the European Spine Society, the European Spinal Deformity Society, and the European Section of the Cervical. Spine Research Society. 2016;25(11):3411-7. doi:10.1007/s00586-015-3923-0.

10. He D, Yu W, Chen Z, Li L, Zhu K, Fan S. Pathogenesis of the intravertebral vacuum of Kümmell's disease. Experimental therapeutic medicine. 2016;12(2):879-82. doi:10.3892/etm.2016.3369.

11. Saito $S$, Inoue A, Ono K. Intramedullary haemorrhage as a possible cause of avascular necrosis of the femoral head. The histology of 16 femoral heads at the silent stage. The Journal of bone joint surgery British volume. 1987;69(3):346-51. doi:10.1302/0301-620x.69b3.3584182.

12. Kim YC, Kim YH, Ha KY. Pathomechanism of intravertebral clefts in osteoporotic compression fractures of the spine. The spine journal: official journal of the North American Spine Society. 2014;14(4):659-66. doi:10.1016/j.spinee.2013.06.106.

13. Lin WC, Chen HL, Lu CH, Wang HC, Wu RW, Cheng YF, Lui CC. Dynamic contrast-enhanced magnetic resonance imaging for evaluating intraosseous cleft formation in patients with osteoporotic vertebral compression fractures before vertebroplasty. Spine. 2011;36(15):1244-50.

doi:10.1097/BRS.0b013e3181eb9b6c.

14. Yu CW, Hsu CY, Shih TT, Chen BB, Fu CJ. Vertebral osteonecrosis: MR imaging findings and related changes on adjacent levels. AJNR American journal of neuroradiology. 2007;28(1):42-7.

15. Karasick D, Eason MA. Vertebral pneumatocyst mimicking susceptibility artifact on MR imaging. AJR American journal of roentgenology. 1998;170(1):221. doi:10.2214/ajr.170.1.9423641.

16. Theodorou DJ. The intravertebral vacuum cleft sign. Radiology. 2001;221(3):787-8. doi:10.1148/radiol.2213991129. 
17. Pesce V, Piazzolla A, Moretti L, Carlucci S, Parato C, Maxy P, Moretti B. The vertebral biomechanic previous and after kyphoplasty. Aging clinical experimental research. 2013;25(Suppl 1):71-4. doi:10.1007/s40520-013-0073-2.

18. Hasegawa K, Homma T, Uchiyama S, Takahashi H. Vertebral pseudarthrosis in the osteoporotic spine. Spine. 1998;23(20):2201-6. doi:10.1097/00007632-199810150-00011.

19. Yu W, Liang D, Yao Z, Qiu T, Ye L, Huang X, Jiang X. Risk factors for recollapse of the augmented vertebrae after percutaneous vertebroplasty for osteoporotic vertebral fractures with intravertebral vacuum cleft. Medicine. 2017;96(2):e5675. doi:10.1097/md.0000000000005675.

20. Ratcliffe JF. The arterial anatomy of the adult human lumbar vertebral body: a microarteriographic study. Journal of anatomy. 1980;131(Pt 1):57-79.

21. Prakash, Prabhu LV, Saralaya VV, Pai MM, Ranade AV, Singh G, Madhyastha S. (2007) Vertebral body integrity: a review of various anatomical factors involved in the lumbar region. Osteoporosis international: a journal established as result of cooperation between the European Foundation for Osteoporosis and the National Osteoporosis Foundation of the USA 18 (7):891-903. doi:10.1007/s00198-007-0373-5.

22. Yu W, Xu W, Jiang X, Liang D, Jian W. Risk Factors for Recollapse of the Augmented Vertebrae After Percutaneous Vertebral Augmentation: A Systematic Review and Meta-Analysis. World neurosurgery. 2018;111:119-29. doi:10.1016/j.wneu.2017.12.019.

23. Li Z, Liu T, Yin P, Wang Y, Liao S, Zhang S, Su Q, Hai Y. The therapeutic effects of percutaneous kyphoplasty on osteoporotic vertebral compression fractures with or without intravertebral cleft. International orthopaedics. 2019;43(2):359-65. doi:10.1007/s00264-018-4007-7.

24. Fang X, Yu F, Fu S, Song H. Intravertebral clefts in osteoporotic compression fractures of the spine: incidence, characteristics, and therapeutic efficacy. Int J Clin Exp Med. 2015;8(9):16960-8.

25. Yu W, Liang D, Yao Z, Qiu T, Ye L, Jiang X. The therapeutic effect of intravertebral vacuum cleft with osteoporotic vertebral compression fractures: A systematic review and meta-analysis. International journal of surgery (London England). 2017;40:17-23. doi:10.1016/j.jjsu.2017.02.019.

26. Lee SH, Kim ES, Eoh W. Cement augmented anterior reconstruction with short posterior instrumentation: a less invasive surgical option for Kummell's disease with cord compression. Journal of clinical neuroscience: official journal of the Neurosurgical Society of Australasia. 2011;18(4):509-14. doi:10.1016/j.jocn.2010.07.139.

27. Chen L, Dong R, Gu Y, Feng Y. Comparison between Balloon Kyphoplasty and Short Segmental Fixation Combined with Vertebroplasty in the Treatment of Kümmell's Disease. Pain Physician. 2015;18(4):373-81.

28. Huang YS, Hao DJ, Feng H, Zhang HP, He SM, Ge CY, Niu XB. Comparison of Percutaneous Kyphoplasty and Bone Cement-Augmented Short-Segment Pedicle Screw Fixation for Management of Kümmell Disease. Medical science monitor: international medical journal of experimental clinical research. 2018;24:1072-9. doi:10.12659/msm.905875. 


\section{Figures}
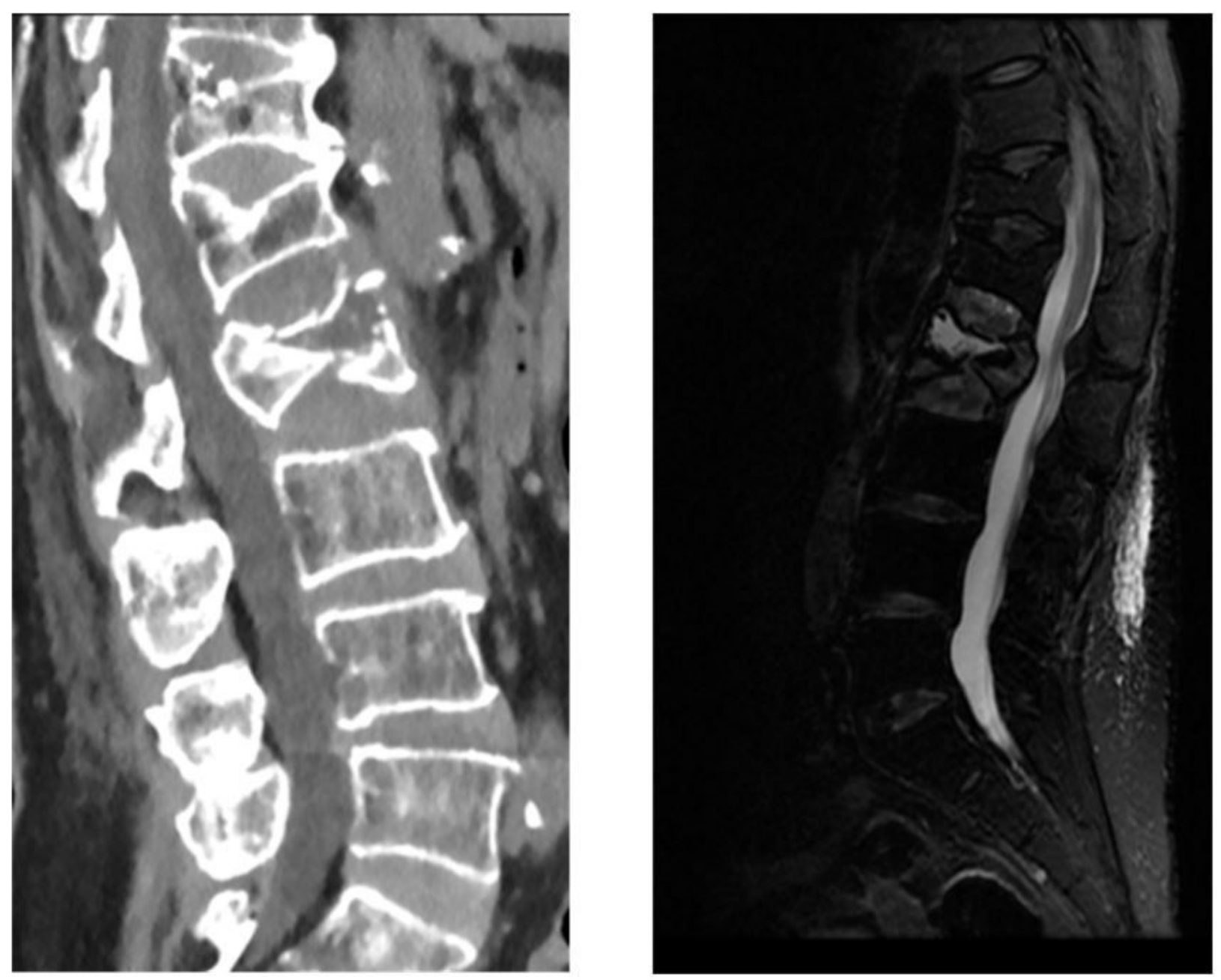

\section{Figure 1}

Characteristic radiological imaging of KD. An 82-year-old female who had low back pain when she woke up 20 days ago. She visited the emergency department on the same day, X-ray examination revealed old VCF of T12 and L1 (past medical history). Later, the symptoms gradually worsened, and reexamination of X-ray revealed a fresh VCF of L2. Further CT and MR examinations revealed a new VCFs of L2 and IVC formation. 

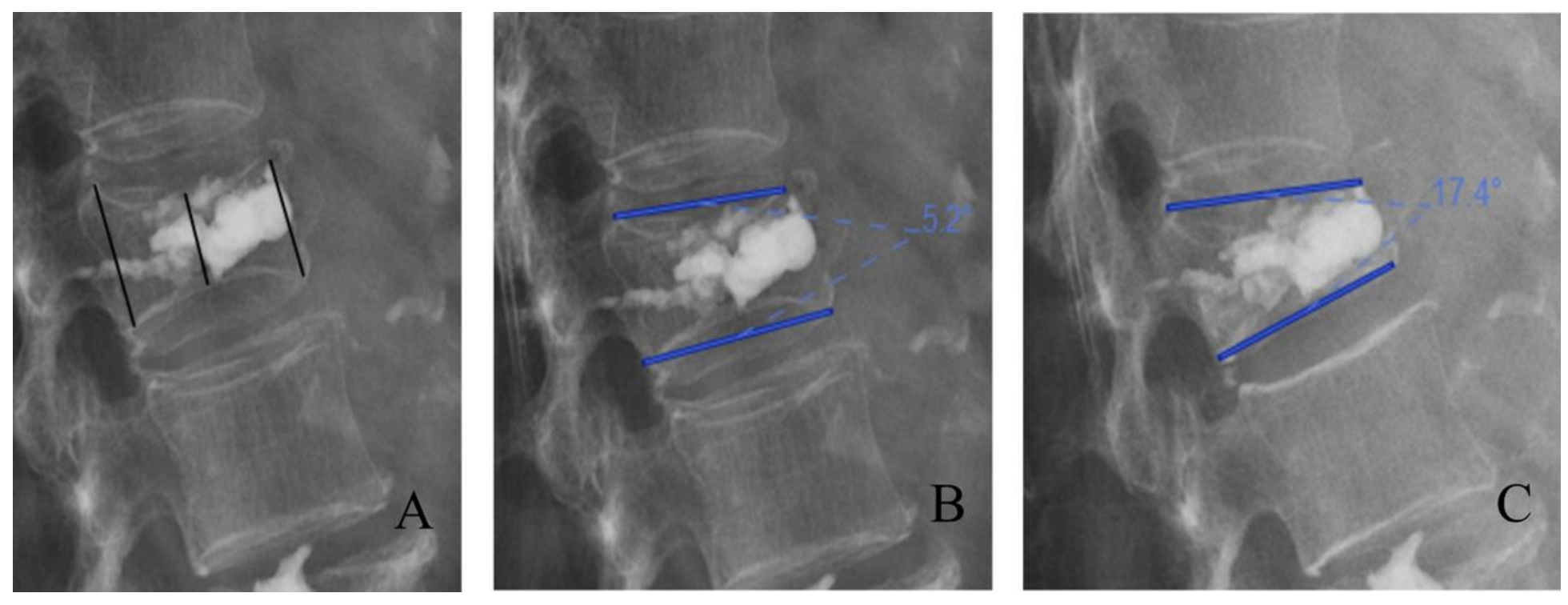

\section{Figure 2}

Legends for calculating vertebral compression rate (VCR) and kyphotic angle (KA). Figure A shows the illustration of VCR. VCR $=1$ - the height of the maximum compression of the vertebral body*2/ (anterior edge height of vertebral body + posterior edge height of vertebral body). Figure B shows the illustration of the KA. The KA is defined as the angle formed between the cranial endplates and the caudal endplates of the vertebral body. Figure $\mathrm{C}$ shows the reexamination results of the same vertebral body after 1 year, the progress of KA is greater than $10^{\circ}$, which meets the diagnostic criteria of recollapse. 


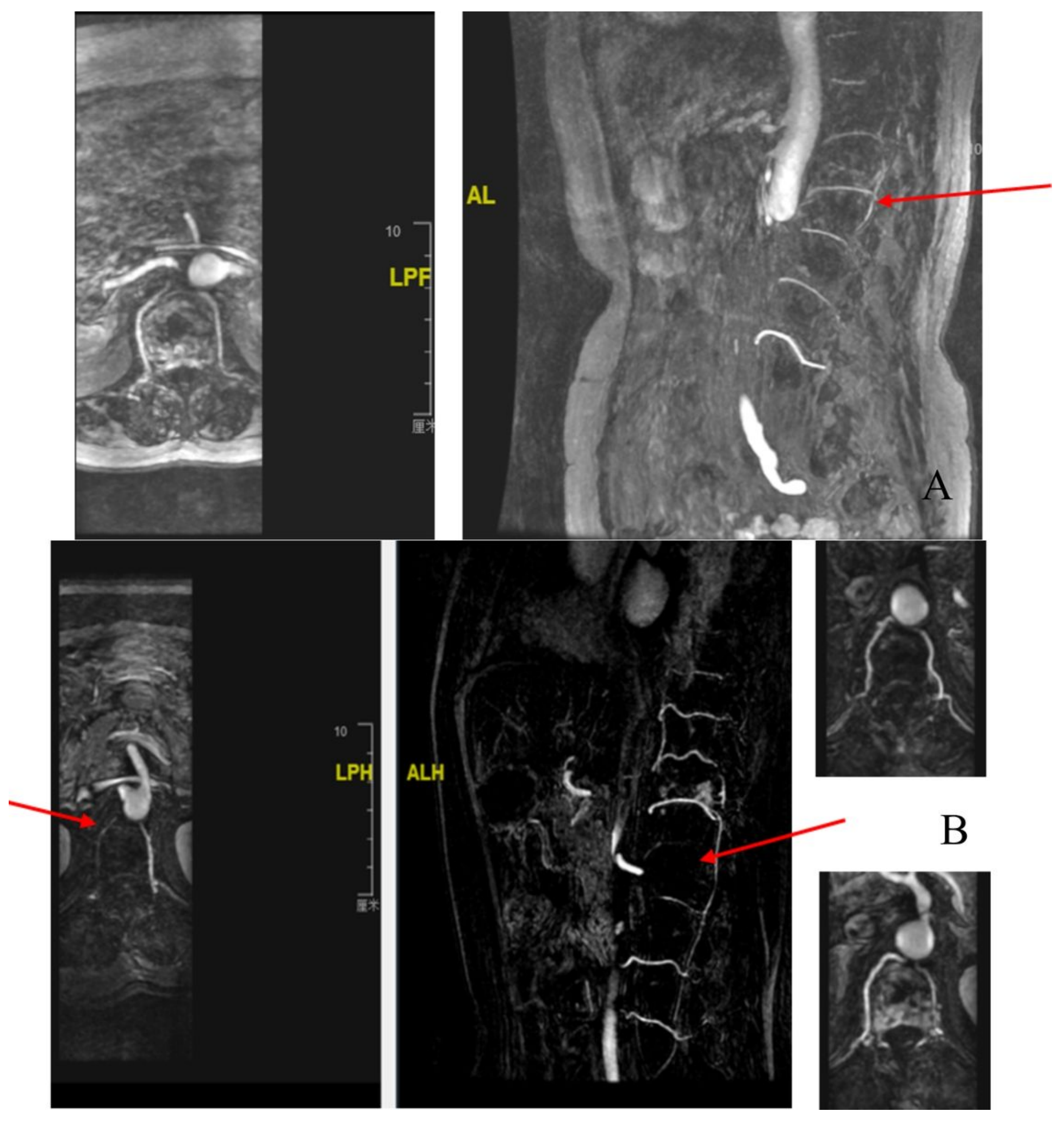

Figure 3

Typical imaging of MRA. Figure A shows the MRA results of compression fractured vertebral body (L1) from the sagittal and horizontal planes: bilateral segmental arteries are expedite. Figure $B$ shows the MRA results of compression fractured vertebral body (L1) from the sagittal and horizontal planes: the artery of the right segment is blocked, and is a significant difference between the imaging results of the MRA of the adjacent vertebral body. 